\title{
Postpartum diagnosis demonstrates a high risk for metastasis and merits an expanded definition of pregnancy-associated breast cancer
}

\author{
Eryn B. Callihan • Dexiang Gao - Sonali Jindal - Traci R. Lyons • \\ Elizabeth Manthey $\cdot$ Susan Edgerton - Alexander Urquhart • \\ Pepper Schedin • Virginia F. Borges
}

Received: 2 November 2012/ Accepted: 28 January 2013/Published online: 22 February 2013

(C) The Author(s) 2013. This article is published with open access at Springerlink.com

\begin{abstract}
Previous studies report conflicting data on outcomes of pregnancy-associated breast cancer (PABC). Our aim was to examine the effect of a postpartum diagnosis on maternal prognosis in a young women's breast cancer cohort. We conducted a retrospective cohort study of women age $\leq 45$ years, diagnosed with breast cancer $(n=619)$ during 1981-2011 at the University of Colorado Hospital and The Shaw Cancer Center in Edwards, CO. Breast cancer cases were grouped according to time between giving birth and diagnosis: nulliparous $(n=125)$, pregnant $(n=24),<5$ years postpartum $(n=136),>5-$ $<10$ postpartum $(n=130)$, and $\geq 10$ years postpartum
\end{abstract}

Electronic supplementary material The online version of this article (doi:10.1007/s10549-013-2437-x) contains supplementary material, which is available to authorized users.

E. B. Callihan - S. Jindal - T. R. Lyons - E. Manthey ·

P. Schedin · V. F. Borges $(\square)$

Young Women's Breast Cancer Translational Program,

University of Colorado Cancer Center, University of Colorado

Denver Anschutz Medical Campus, 12801 East 17th Avenue

MS 8117, RM 8112, Aurora, CO 80045, USA

e-mail: virginia.borges@ucdenver.edu

E. B. Callihan - S. Jindal - T. R. Lyons - E. Manthey ·

P. Schedin · V. F. Borges

Division of Medical Oncology, University of Colorado Denver

Anschutz Medical Campus, 12801 E 17th Ave, RC1 South,

MS 8117, Aurora, CO 80045, USA

D. Gao

Department of Pediatrics, University of Colorado Denver

Anschutz Medical Campus, Aurora, CO, USA

D. Gao

Department of Biostatistics and Informatics, Colorado School of Public Health, University of Colorado Denver Anschutz Medical Campus, 12477 East 19th Avenue Bldg 406, Room 105, Aurora, CO 80045, USA
( $n=147)$, to examine the clinicopathologic features and the risk of distance recurrence and death. Cases diagnosed after pregnancy, but within five-years postpartum, had an approximate three fold increased risk of distant recurrence (HR 2.80, $95 \%$ CI: 1.12-6.57) and death (HR 2.65, $95 \%$ CI: 1.09-6.42) compared to nulliparous cases. Postpartum cases diagnosed within five years of last childbirth demonstrated a higher five-year distant recurrence probability $(31.1 \%)$ and a markedly lower five-year overall survival probability $(65.8 \%)$ compared to nulliparous cases (14.8 and $98.0 \%$, respectively). A diagnosis of breast cancer during the first five-years postpartum confers poorer maternal prognoses after adjustment for biologic subtype, stage, and year of diagnosis. We propose that the definition of PABC should include cases diagnosed up to at least five-

\section{S. Edgerton}

Department of Pathology, University of Colorado Denver Anschutz Medical Campus, 12801 East 17th Avenue, PO Box 8104, Aurora, CO 80045, USA

\section{A. Urquhart}

Medical Oncology, Shaw Regional Cancer Center, University of Colorado Cancer Center, Beard Creek Road, PO Box 2559, Edwards, CO 81632, USA

P. Schedin

Program in Cancer Biology, University of Colorado Denver Anschutz Medical Campus, Aurora, CO 80045, USA 
years postpartum to better delineate the increased risk imparted by a postpartum diagnosis. Based on emerging preclinical and epidemiologic data, we propose that pregnant and postpartum cases be researched as distinct subsets of PABC to clarify the risk imparted by pregnancy and the events subsequent to pregnancy, such as breast involution, on breast cancer. Further, we highlight the importance of postpartum breast cancer as an area for further research to reduce the increased metastatic potential and mortality of PABC.

Keywords Pregnancy-associated breast cancer . Young women's breast cancer - Postpartum breast cancer . Survival · Metastasis

$\begin{array}{ll}\text { Abbreviations } \\ \text { PABC } & \text { Pregnancy-associated breast cancer } \\ \text { HR } & \text { Hazard ratio } \\ \text { pHR } & \text { Pooled hazard ratio } \\ \text { DR } & \text { Distant recurrence } \\ \text { OS } & \text { Overall survival }\end{array}$

\section{Background}

Breast cancer is the leading US cancer diagnosis in women age 20-45 and is the leading malignancy diagnosed in association with pregnancy in the US [1-3]. Pregnancy results in a complex " "dual effect" on breast cancer risk [4] with a transient increased risk of breast cancer diagnosis observed during the subsequent postpartum years for all first time mothers [5-7]. This increased risk is reported to last up to 15 years after childbirth regardless of the mother's age [5]. For women who are under 35 years at first birth, a "cross-over" effect eventually begins to provide long-term protection against breast cancer [8-10]. Thus, the effect of a prior pregnancy on the risk for a young women's breast cancer risk is complex, from initial promotion in the postpartum period to one of protection years to decades later.

A pregnancy-associated breast cancer (PABC) is a breast cancer diagnosed during or a few years subsequent to a recent pregnancy. The specific definition of PABC has varied throughout the literature and this variability in defining PABC has led to conflicting results on the relationship between pregnancy, prior pregnancy and breast cancer outcomes. PABC is often limited to breast cancer diagnosed during pregnancy or within one-year postpartum [1, 11-16]. A number of studies using this definition demonstrate PABC as having inferior prognosis $[1,15,16$, $18,21]$, while two studies identify no increased mortality
[11, 12]. Conversely, five large epidemiologic studies specifically isolating postpartum breast cancers uniformly report poor maternal outcomes associated with postpartum PABC [22-26]. A recently published meta-analysis of 30 PABC studies further suggest that mothers diagnosed postpartum have a significantly worse overall survival (OS) (pHR 1.84, $95 \%$ CI: 1.2-2.65) compared to non-PABC breast cancer cases [21]. Thus, when PABC is defined as combined pregnant and early postpartum cases, the epidemiologic data on maternal prognosis is inconsistent. However, when cases diagnosed postpartum are examined separately from cases diagnosed during pregnancy, the data demonstrate an increased and potentially prolonged maternal risk of poor outcomes, suggesting an expanded definition of PABC is warranted.

The convention of limiting PABC to pregnant and early postpartum diagnoses originated from studies demonstrating tumor growth promotion by hormonal milieus similar to those occurring during pregnancy $[10,27,28]$. However, in preclinical models, we reported that during normal, postpartum mammary gland involution, the process where the tissue remodels from a lactation competent to a nonlactational state, the gland acquires several attributes in common with wound-healing and tumor desmoplastic microenvironments [29]. Furthermore, we have demonstrated that postpartum involution increases the metastatic potential of human tumor cells in xenograft models of postpartum breast cancer [30]. Based on these observations, we proposed that postpartum involution is a unique biologic event, separate from the effects of pregnancy, that may contribute to the poor prognosis of postpartum breast cancer [30].

In this study, we characterized young women's breast cancer by luminal A, luminal B, Her2 neu positive, and triple negative biologic subtypes and other clincopathologic features. In addition, we examined the association of distant recurrence (DR) and OS with time since last child birth to delineate the relationship between a postpartum diagnosis and maternal prognosis.

\section{Methods}

A retrospective cohort study was conducted with 619 women age $\leq 45$ years diagnosed with breast cancer during 1981-2011 at University of Colorado Hospital $(n=539)$ and The Shaw Cancer Center in Edwards, CO $(n=80)$. Patient data were abstracted through medical chart review. $71 \%$ of cases are self-reported Caucasian $(n=440), 9 \%$ Hispanic $(n=56), 4.5 \%$ Black $(n=28)$, and $3.7 \%$ other $(n=23) .11 .8 \%(n=72)$ chose not to report racial demographic information. All research was approved by the Colorado Multiple Institutional Review Board. 
Defining cases by parity status

Postpartum breast cancer was defined as cancers diagnosed within five years of a woman's last childbirth (PABC $<5$, $n=136$ ), and those diagnosed during pregnancy were considered a separate subset of PABC $(n=24)$. All cases in the pregnant subset completed delivery of the co-diagnosed child. Later parous cases were combined according to years between last childbirth and breast cancer diagnosis: parous $>5-<10(n=130)$ and parous $\geq 10(n=147)$. Nulliparous women with no evidence of previous pregnancy were used as the referent population $(n=125)$. Nulliparous women with evidence of incomplete pregnancy were excluded $(n=57)$.

Clinical characterization of young women breast cancer

Cases were characterized according to parity grouping ( $n=562$ ). Clinical stage was defined according to the AJCC Cancer Staging Manual (7th Edition). Luminal A biologic subtype was defined as $\mathrm{ER}+, \mathrm{PR}+, \mathrm{Her} 2$ neu negative; luminal $\mathrm{B}$ defined as $\mathrm{ER}+, \mathrm{PR} \pm$, Her2 neu+; Her2 neu+ defined by ER-, PR - , Her2 neu+; and a triple negative defined by ER, PR and Her2 neu all negative .

\section{Statistical analysis}

Cohort demographic, clinical, and treatment data are summarized in Table 1. To test for intergroup differences, Chi-square and Fishers Exact Test (categorical variables), and One-Way ANOVA (continuous variables) were used.

Estimating risk of distant recurrence (DR) and overall survival (OS)

A DR was defined as the presentation of breast cancer metastasis following completed primary breast cancer staging. Cases with no evidence of DR and cases alive at the end of followup were censored at the date of last contact. To better power our analysis, cases diagnosed $\geq 5$ years following last childbirth were collapsed. Nulliparous cases with evidence of prior pregnancy $(n=57)$ and pregnant cases ( $n=24)$ were excluded from the outcomes analyses. Kaplan-Meier curves were created to determined crude differences in DR and OS. Cox proportional multivariate regression models were performed to obtain adjusted risk estimates for DR, OS, hazard ratios and $95 \%$ confidence intervals. Cases missing followup data $(n=52)$, model covariates ( $n=189)$, declining treatment $(n=4)$, or having multiple primary breast cancers $(n=6)$, and cases stage 0 $(n=15)$ and stage IV $(n=27)$ were excluded. After these exclusions, 326 cases were analyzed for DR. Stage IV cases were included in the OS analysis to reflect true population outcomes, resulting in 334 cases available for the OS analysis. Stage, biologic subtype, prior local recurrence, and year of diagnosis were included in the final multivariate model of DR. Stage, biologic subtype, and year of diagnosis were included in the final multivariate model of OS. Age at diagnosis was not significantly different between groups and was not included in the multivariate models.

All statistics were performed using SAS 9.2. A $p$ value of $\leq 0.05$ was considered statistically significant.

\section{Results}

Table 1 shows the clinical characterization of our Colorado young women's breast cancer cohort. We did not observe a significant difference in histologic subtype across parity groups $(p=0.09)$. While there was a slightly higher frequency of lobular carcinoma in all parous groups compared to nulliparous, this result was not significant. Similarly, we did not detect a significant difference in histologic grade $(p=0.27)$, tumor size $(p=0.18)$, clinical stage $(p=0.40)$, or nodal involvement $(p=0.45)$ between parity groups. While all postpartum groups demonstrated an increased frequency of lymphovascular invasion compared to nulliparous, these differences were not significant. We did not observe differences in chemotherapy and radiation treatment modalities between parity groups. However, compared to $\mathrm{PABC}<5$ cases, nulliparous cases more often underwent breast conservation therapy (22.9 vs. $46.8 \%$ ), while PABC $<5$ cases received mastectomy more frequently compared to nulliparous cases $(p<0.0001)$ (Table 1$)$.

To evaluate the effect of prior pregnancy on maternal outcomes, we examined the risk of DR and OS. Postpartum PABC cases demonstrated a higher five-year DR probability (31.1\%) compared to nulliparous cases $(14.8 \%$ ) (Fig. 1a, b) and had a significant 2.8 times higher risk of DR (HR 2.80, 95 \% CI: 1.12-6.57) compared to nulliparous cases (Table 2). The multivariate DR risk model demonstrated an increased metastatic potential associated with a breast cancer diagnosed within five-years postpartum, adjusting for stage at diagnosis, biologic subtype, prior local recurrence, and year of diagnosis.

$\mathrm{PABC}<5$ cases demonstrated a crude lower five-year OS probability $(65.8 \%)$ compared to nulliparous cases $(98.0 \%)$ (Fig. 1c, d). The relatively high five-year OS in this nulliparous cohort mirrors previously published data [18]. After adjusting for stage, biologic subtype, and year of diagnosis, PABC $<5$ cases had a significant 2.7 times greater risk of death compared to nulliparous cases (HR 2.65, $95 \%$ CI: 1.09-6.42) (Table 3).

To evaluate clinical outcomes for cases diagnosed $\geq 5$-years postpartum, we compressed later parity cohorts to increase statistical power (Supplemental Table 8). Our data 
Table 1 Clinical Characterization of UC Young Women's Breast Cancer Cohort

\begin{tabular}{|c|c|c|c|c|c|c|}
\hline \multirow[t]{2}{*}{ Parity group } & \multirow{2}{*}{$\begin{array}{l}\text { Nulliparous } \\
(N=125) \\
\text { No. }(\%)\end{array}$} & \multirow{2}{*}{$\begin{array}{l}\mathrm{PABC}<5 \\
(N=136) \\
\text { No. }(\%)\end{array}$} & \multirow{2}{*}{$\begin{array}{l}\text { Parous } \geq 5-<10 \\
(N=130) \\
\text { No. }(\%)\end{array}$} & \multirow{2}{*}{$\begin{array}{l}\text { Parous } \geq 10 \\
(N=147) \\
\text { No. }(\%)\end{array}$} & \multicolumn{2}{|l|}{$p$ value } \\
\hline & & & & & Overall & $\begin{array}{l}\mathrm{PABC}<5 \text { vs. } \\
\text { nulliparous }\end{array}$ \\
\hline Mean age at diagnosis & $37.3 \pm 5.5$ & $35.7 \pm 5.3$ & $38.7 \pm 4.5$ & $40.9 \pm 3.5$ & $<0.0001^{\mathrm{d}}$ & $<0.05^{\mathrm{d}}$ \\
\hline \multicolumn{7}{|l|}{ Histologic subtype } \\
\hline Ductal & $109(87.20)$ & $120(88.24)$ & $111(85.38)$ & $119(80.95)$ & \multirow[t]{6}{*}{$0.09^{\mathrm{e}}$} & \multirow[t]{6}{*}{$0.54^{\mathrm{e}}$} \\
\hline Lobular & $1(0.80)$ & $5(3.68)$ & $10(7.69)$ & $12(8.16)$ & & \\
\hline Ductal + lobular & $3(2.40)$ & $3(2.21)$ & $5(3.85)$ & $6(4.08)$ & & \\
\hline Inflammatory & $2(1.60)$ & $2(1.47)$ & $1(0.77)$ & $5(3.40)$ & & \\
\hline Other & $5(4.00)$ & $4(2.94)$ & $1(0.77)$ & $2(1.36)$ & & \\
\hline Missing & $5(4.00)$ & $2(1.47)$ & $2(1.54)$ & $3(2.04)$ & & \\
\hline \multicolumn{7}{|l|}{ Biologic subtype } \\
\hline Luminal A & $38(30.40)$ & $52(38.24)$ & $47(36.15)$ & $49(33.33)$ & \multirow[t]{6}{*}{$0.96^{\mathrm{f}}$} & \multirow[t]{6}{*}{$0.78^{\mathrm{f}}$} \\
\hline Luminal B & $18(14.40)$ & $17(12.50)$ & $13(10.00)$ & $18(12.24)$ & & \\
\hline Her2 neu positive & $11(8.80)$ & $11(8.09)$ & $12(9.23)$ & $13(8.84)$ & & \\
\hline Triple negative & $18(14.4)$ & $20(14.71)$ & $16(12.31)$ & $25(17.01)$ & & \\
\hline Missing Her2 neu ${ }^{\mathbf{a}}$ & $28(22.40)$ & $28(20.59)$ & $26(20.00)$ & $32(21.77)$ & & \\
\hline Missing ER or PR & $12(9.60)$ & $8(5.88)$ & $16(12.30)$ & $10(6.80)$ & & \\
\hline \multicolumn{7}{|l|}{ Estrogen status } \\
\hline $\mathrm{ER}+$ & 77 (61.6) & $87(63.9)$ & $81(62.3)$ & $83(56.46)$ & \multirow[t]{3}{*}{$0.37^{\mathrm{f}}$} & \multirow[t]{3}{*}{$0.48^{\mathrm{f}}$} \\
\hline ER- & $39(31.2)$ & $37(27.20)$ & $39(30.0)$ & $54(36.73)$ & & \\
\hline Missing & $9(7.2)$ & $10(7.35)$ & $10(7.69)$ & $10(6.80)$ & & \\
\hline \multicolumn{7}{|l|}{ Histologic grade } \\
\hline Grade I & $15(12.00)$ & $10(7.35)$ & $14(10.77)$ & $18(12.24)$ & \multirow[t]{4}{*}{$0.27^{\mathrm{f}}$} & \multirow[t]{4}{*}{$0.55^{\mathrm{f}}$} \\
\hline Grade II & $39(31.20)$ & $39(28.68)$ & $39(30.00)$ & $60(40.82)$ & & \\
\hline Grade III & $61(48.80)$ & $73(53.68)$ & $63(48.46)$ & $54(36.73)$ & & \\
\hline Missing & $10(8.00)$ & $14(10.29)$ & $14(10.77)$ & $15(10.20)$ & & \\
\hline \multicolumn{7}{|l|}{ Tumor size } \\
\hline $0.1-\leq 2.0 \mathrm{~cm}$ & $61(48.80)$ & $67(49.26)$ & 49 (37.69) & $62(42.18)$ & \multirow[t]{4}{*}{$0.18^{\mathrm{f}}$} & \multirow[t]{4}{*}{$0.97^{\mathrm{f}}$} \\
\hline$>2.0-\leq 5.0 \mathrm{~cm}$ & $41(32.80)$ & $44(32.35)$ & $45(34.62)$ & $59(40.14)$ & & \\
\hline$>5.0 \mathrm{~cm}$ & $14(11.20)$ & $14(10.29)$ & $25(19.23)$ & $20(13.60)$ & & \\
\hline Missing & $9(7.20)$ & $11(8.09)$ & $11(8.46)$ & $6(4.08)$ & & \\
\hline \multicolumn{7}{|l|}{ Stage } \\
\hline 0 & $4(3.20)$ & $3(2.21)$ & $5(3.85)$ & $3(2.04)$ & \multirow[t]{6}{*}{$0.40^{\mathrm{f}}$} & $0.62^{\mathrm{f}}$ \\
\hline I & $36(28.80)$ & $34(25.00)$ & 27 (20.77) & $39(26.53)$ & & \\
\hline II & $52(41.60)$ & $52(38.24)$ & $57(43.85)$ & $62(42.18)$ & & \\
\hline III & $20(16.00)$ & $26(19.12)$ & $31(23.85)$ & $31(21.09)$ & & \\
\hline IV & $6(4.80)$ & $12(8.82)$ & $2(1.54)$ & 7 (4.76) & & \\
\hline Missing & $7(5.60)$ & $9(6.62)$ & $8(6.15)$ & $5(3.40)$ & & \\
\hline Lymph node involver & & & & & & \\
\hline Positive & $59(47.20)$ & $76(55.88)$ & $64(49.23)$ & $71(48.30)$ & $0.45^{\mathrm{f}}$ & $0.14^{\mathrm{f}}$ \\
\hline Negative & $64(51.20)$ & $57(41.91)$ & $65(50.00)$ & $72(48.98)$ & & \\
\hline Missing & $2(1.60)$ & $3(2.21)$ & $1(0.77)$ & $4(2.72)$ & & \\
\hline Lymphovascular inva & & & & & & \\
\hline Present & $28(22.40)$ & $40(29.41)$ & $40(30.77)$ & $57(38.78)$ & $0.21^{\mathrm{f}}$ & $0.15^{\mathrm{f}}$ \\
\hline Absent & $55(44.00)$ & $50(36.76)$ & $53(40.77)$ & $60(40.82)$ & & \\
\hline Missing & $42(33.60)$ & $46(33.82)$ & 37 (28.46) & $30(20.41)$ & & \\
\hline
\end{tabular}


Table 1 continued

\begin{tabular}{|c|c|c|c|c|c|c|}
\hline \multirow[t]{2}{*}{ Parity group } & \multirow{2}{*}{$\begin{array}{l}\text { Nulliparous } \\
(N=125) \\
\text { No. }(\%)\end{array}$} & \multirow{2}{*}{$\begin{array}{l}\text { PABC }<5 \\
(N=136) \\
\text { No. }(\%)\end{array}$} & \multirow{2}{*}{$\begin{array}{l}\text { Parous } \geq 5-<10 \\
(N=130) \\
\text { No. }(\%)\end{array}$} & \multirow{2}{*}{$\begin{array}{l}\text { Parous } \geq 10 \\
(N=147) \\
\text { No. }(\%)\end{array}$} & \multicolumn{2}{|l|}{$p$ value } \\
\hline & & & & & Overall & $\begin{array}{l}\text { PABC }<5 \text { vs. } \\
\text { nulliparous }\end{array}$ \\
\hline \multicolumn{7}{|l|}{ Surgery type ${ }^{b}$} \\
\hline No surgery & $0(0)$ & $3(2.5)$ & $1(0.88)$ & $3(2.3)$ & $<0.0001^{\mathrm{f}}$ & $<0.0001^{\mathrm{f}}$ \\
\hline Local excision $^{c}$ & $0(0)$ & $0(0)$ & $1(0.88)$ & $1(0.8)$ & & \\
\hline Lumpectomy & $51(46.8)$ & $27(22.9)$ & $32(28.0)$ & $36(28.1)$ & & \\
\hline Unilateral mastectomy & $25(22.9)$ & $37(31.4)$ & $48(42.1)$ & $51(39.8)$ & & \\
\hline Bilateral mastectomy & $15(13.8)$ & $37(31.4)$ & $17(14.9)$ & $11(8.6)$ & & \\
\hline Missing & $18(16.5)$ & $14(11.8)$ & $15(13.2)$ & $26(20.3)$ & & \\
\hline \multicolumn{7}{|l|}{ Chemotherapy $^{\mathrm{b}}$} \\
\hline Yes & 66 (60.6) & 79 (67.0) & $71(62.3)$ & $71(55.5)$ & $0.55^{\mathrm{f}}$ & $0.17^{\mathrm{f}}$ \\
\hline No & $22(20.2)$ & $16(13.6)$ & $21(18.4)$ & $22(17.2)$ & & \\
\hline Missing & $21(19.2)$ & $23(19.4)$ & $22(19.3)$ & $35(27.3)$ & & \\
\hline \multicolumn{7}{|l|}{ Radiation therapy $^{\mathrm{b}}$} \\
\hline Yes & $57(52.2)$ & $51(43.2)$ & $61(53.5)$ & $50(39.0)$ & $0.07^{\mathrm{f}}$ & $0.22^{\mathrm{f}}$ \\
\hline No & $29(26.6)$ & $38(32.2)$ & $26(22.8)$ & $44(34.4)$ & & \\
\hline Missing & $23(21.1)$ & $29(24.6)$ & $27(23.7)$ & $34(26.6)$ & & \\
\hline
\end{tabular}

${ }^{a}$ Missing Her2 neu data, reflecting unavailable Her2 neu staining or FISH analysis at diagnosis, was evenly distributed across parity groups and thus is not anticipated to confound results

b Treatment data for cases diagnosed at the University of Colorado Hospital ( $n=469)$-nulliparous $(n=109)$, PABC $<5$ ( $N=118)$, Parous $\geq 5-<10(n=114)$; Parous $\geq 10(n=128)$. Treatment data unavailable for cases diagnosed at The Shaw Cancer Center $(n=80)$; treatment data for cases diagnosed during pregnancy are included in supplemental Table 7

c Primary unknown

d One Way Anova with Tukey's Multiple Comparisons Test

e Fisher's Exact Test

${ }^{\mathrm{f}} \chi^{2}$ Test

suggest that cases diagnosed $\geq 5$-years postpartum may have a prognosis intermediate to $\mathrm{PABC}<5$ and nulliparous cases (Fig. 1a-d). These cases demonstrated a crude $19.9 \%$ fiveyear DR probability and crude $77.5 \%$ five-year OS probability. Cases diagnosed $\geq 5$ had a significantly lower DR risk compared to PABC $<5$ cases (HR 0.36; $95 \%$ CI: 0.18-0.75) (Table 2). However, compared to nulliparous cases, cases $\geq 5$ postpartum showed a trend toward higher risk of death (HR 1.52; $95 \%$ CI: 0.71-3.28), but this result did not meet significance (Table 3). Age at diagnosis was examined as both a continuous and categorical variable $(<35$ or $\geq 35$, or $<40$ or $\geq 40$ ) in the univariate DR and OS models, but did not reach statistical criterion for inclusion in the final models. Hazard ratios for the final multivariate models are included in the supplemental material (Table 6, Table 7).

To investigate whether PABC may be two distinct subsets, based on patient outcomes, we combined cases based on previously published definitions [1, 11, 12, 15, 16, 18], and grouped pregnant cases and cases diagnosed $\leq 1$ year postpartum as in the "PABC group." Nulliparous and/or parous women diagnosed $>1$ year postpartum were combined to comprise the "non-PABC" controls, mirroring methods used in two recent studies reporting negative results $[11,12]$. When grouped this way, we also did not observe a significant difference in OS between "PABC" and "nonPABC" cases (Table 4). To examine if the poor prognosis observed in our PABC $<5$ cohort is driven by cases diagnosed early postpartum $(\leq 1)$, we examined the adjusted OS in postpartum $\leq 1$ compared to postpartum $>1-5$. We did not observe a significantly lower OS in postpartum cases $\leq 1$ compared to the later postpartum cases $(p=0.51)$. Further, when we examined the crude number of deaths broken down by years postpartum at diagnosis $[\leq 1,>1-\leq 2,>2-\leq 3$, $>3-\leq 4$, and $>4-\leq 5$ years], we did not observe a skewing of deaths to the earlier postpartum time points (Fig. 2).

Cases diagnosed during pregnancy showed a high frequency of ductal carcinoma $(91.7 \%)$ and poorly differentiated tumors $(70.8 \%)$. Over half of these pregnant cases had involved lymph nodes $(62.5 \%)$ and $42 \%$ were diagnosed with late stage disease. Luminal A (20\%), Luminal B $(20 \%)$, Her2 neu+ $(20 \%)$, and triple negative $(25 \%)$ biologic subtypes were present in almost equal frequencies (Table 5). However, due to the small number of pregnant 
Fig. 1 a Unadjusted probability of distant recurrence in PABC $<5, \geq 5$, and nulliparous cases demonstrates an increased risk of distant recurrence in postpartum PABC. b Adjusted probability of distance recurrence in $\mathrm{PABC}<5, \geq 5$, and nulliparous cases adjusted for biologic subtype, clinical stage, year of diagnosis, and local recurrence. The adjusted recurrence probability function based on the Cox model was generated for each subject. c Unadjusted overall survival probability in $\mathrm{PABC}<5, \geq 5$ and nulliparous cases demonstrates an increased risk of death in postpartum PABC. d Adjusted overall survival probability in $\mathrm{PABC}<5, \geq 5$, and nulliparous cases adjusted for biologic subtype, clinical stage, and year of diagnosis. The adjusted survival function based on the Cox model was generated for each subject
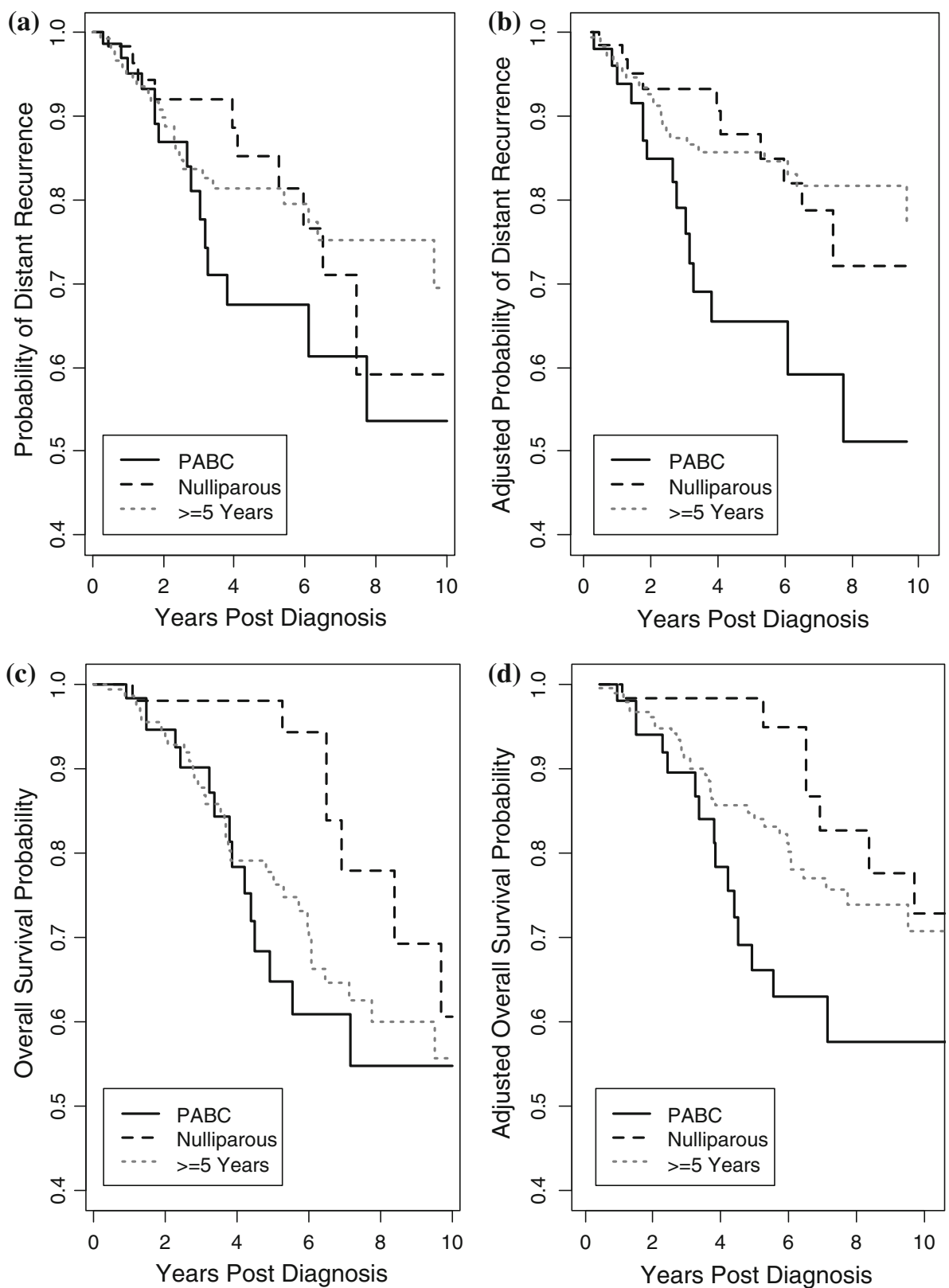

cases $(n=24)$, we did not test for a significant enrichment in these clinical characteristics over non-pregnant cases. Further, due to the small sample size and short followup time, we did not analyze maternal outcomes specific to diagnosis during pregnancy.

In summary, these data demonstrate poor prognosis in breast cancer patients diagnosed within five years of a pregnancy and suggest an intermediate risk in women diagnosed beyond five-years postpartum. When we apply these definitions to this Colorado cohort, we identify of a large subset (29\%) of young breast cancer cases with a potential high risk of DR and death due to recent pregnancy (postpartum $<5$ years) (Fig. 3)

\section{Discussion}

Our results demonstrate that a breast cancer diagnosed within five-years postpartum has a significant 2.8 times higher risk for metastasis and a 2.7 times higher mortality risk compared to nulliparous cases. Our data further shows that a postpartum breast cancer diagnosis is an independent risk factor for recurrence and death. In our cohort, the increased risk of death imparted by a postpartum diagnosis was higher when compared with previous published studies [22-24, 26, 31]. A strength of our study is the ability to include clinical and pathologic characteristics known to affect prognosis into our adjusted outcomes analysis for the 
Table 2 Adjusted risk estimates of distant recurrence

\begin{tabular}{llllll}
\hline Multivariate analysis & & & & \\
\hline Parity group $^{\mathrm{a}}$ & Number of cases & Adjusted $\mathrm{HR}^{\mathrm{b}, \mathrm{c}}$ & $95 \% \mathrm{CI}$ & $p$ & $\begin{array}{l}\text { 5-Year distant recurrence } \\
\text { probability }(\%)\end{array}$ \\
\hline PABC $<5$ & $N=84$ & 2.80 & $1.12-6.57$ & 0.02 & 31.3 \\
Parous $\geq 5$ & $N=168$ & 1.01 & $0.48-2.15$ & 0.97 & 19.9 \\
Nulliparous & $N=74$ & 1.0 & - & - & 14.8 \\
\hline
\end{tabular}

Hazard ratios and $95 \%$ confidence intervals for all covariates included in the final model are described in Table 6 in the supplemental material. Clinical characteristics of the collapsed Parous $\geq 5$ group can be viewed in Table 8 in the supplemental material

Parous $\geq 5$, cases diagnosed five years or later from last childbirth

${ }^{\mathrm{a}} \mathrm{PABC}<5$ cases had a mean followup time of $2.7 \pm 2.7$ years. Mean followup time for nulliparous was $3.2 \pm 3.2$ years and $3.5 \pm 3.6$ years for cases diagnosed $\geq 5$

b Adjusted for tumor biologic subtype, clinical stage, year of diagnosis, and local recurrence

${ }^{c}$ Histologic subtype and grade were not univariately significant and were thus not included in the final multivariate models

Table 3 Adjusted risk estimates of overall survival

\begin{tabular}{llllll}
\hline Multivariate analysis & & & & \\
\hline Parity group $^{\mathrm{a}}$ & Number of cases & Adjusted HR $^{\mathrm{b}, \mathrm{c}}$ & 95 \% CI & $p$ & 5-Year survival (\%) \\
\hline PABC $<5$ & $N=86$ & 2.65 & $1.09-6.42$ & 0.03 & 65.8 \\
Parous $\geq 5$ & $N=172$ & 1.52 & $0.71-3.28$ & 0.28 & 77.5 \\
Nulliparous & $N=76$ & 1.0 & - & - & 98.0 \\
\hline
\end{tabular}

Hazard ratios and $95 \%$ confidence intervals for all covariates included in the final model are described in Table 7 in the supplemental material. Clinical characteristics of the collapsed Parous $\geq 5$ group can be viewed in Table 8 in the supplemental material

Parous $\geq 5$ cases diagnosed five years or later from last childbirth

${ }^{\mathrm{a}} \mathrm{PABC}<5$ cases had a mean followup time of $3.0 \pm 2.9$ years. Mean followup time for nulliparous was $3.6 \pm 3.6$ years and $3.9 \pm 3.7$ years for cases diagnosed $\geq 5$

${ }^{\mathrm{b}}$ Adjusted for tumor biologic subtype, clinical stage, and year of diagnosis

c Histologic subtype and grade were not univariately significant and were thus not included in the final multivariate models

effect of pregnancy on recurrence and death. As such, one potential reason for our higher risk differences may be the absence of clinical characteristics in prior PABC studies and thus the inability to report adjusted risk estimates [23, $26,31]$.

In our cohort, parous women diagnosed $\geq 5$ years following last childbirth trended toward a prognosis intermediate to $\mathrm{PABC}<5$ and nulliparous women. We propose that the period of postpartum risk may persist to at least five-years postpartum as demonstrated by these data, which are likewise supported by previously published studies highlighting the postpartum window of risk [22-26, 31]. In two of these population-based studies, poor outcomes were observed in women diagnosed up to eight years after childbirth [23], as well as a recent study demonstrated peak mortality in women diagnosed postpartum and persisting

Table 4 Adjusted risk estimate of overall survival using published definitions of PABC and control cohorts

\begin{tabular}{llll}
\hline Parity group & Adjusted $\mathrm{HR}^{\mathrm{a}}$ & $95 \%$ CI & $p$ \\
\hline PABC $\leq 1(n=33)$ & 0.78 & $0.29-2.11$ & 0.63 \\
Non-PABC $(\mathrm{A})(n=318)$ & 1.0 & - & - \\
PABC $\leq 1(n=33)$ & 0.77 & $0.28-2.08$ & 0.60 \\
Non-PABC $(\mathrm{B})(n=242)$ & 1.0 & - & - \\
\hline
\end{tabular}

PABC $\leq 1$, cases diagnosed during pregnancy or up to one-year postpartum; Non-PABC (A), nulliparous cases and parous cases diagnosed $>1$ year from last childbirth. Non-PABC (B) 'parous cases diagnosed $>1$ year from last childbirth (nulliparous excluded)

a Adjusted for tumor biologic subtype, clinical stage and year of diagnosis 


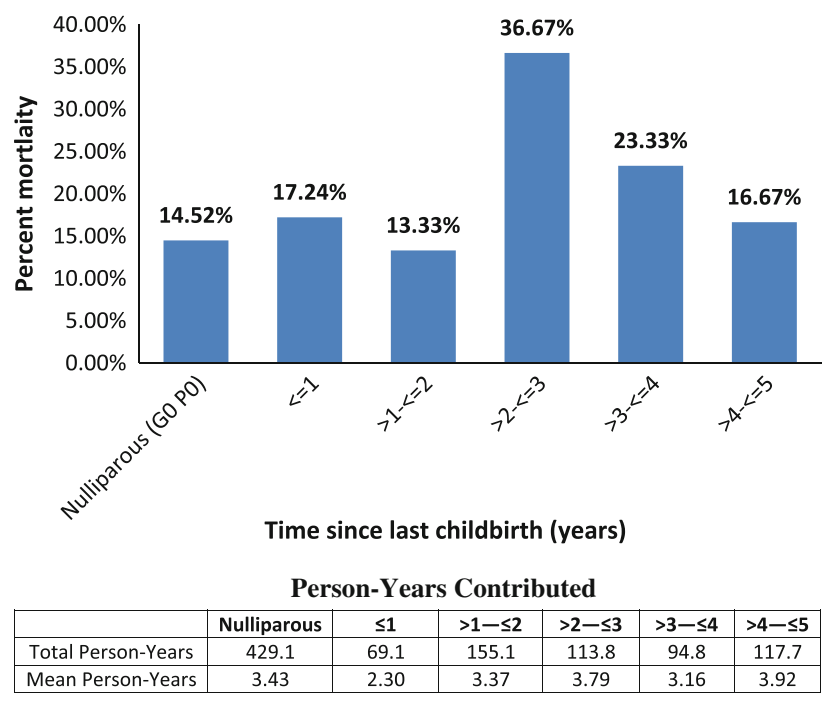

Fig. 2 Crude mortality broken out into years between last childbirth and breast cancer diagnosis shows increased risk continues 3-5 years postpartum

up to 10-years post-diagnosis [26]. We intentionally looked at our data by individual number of years postpartum to identify if an early "cutoff" of increased risk existed and found that the postpartum risk of recurrence and death is not limited to the first or second-year postpartum, as some studies previously suggested $[1,15,16,18]$. At present, we cannot delineate the exact outer limit of this postpartum timeframe which may influence poor maternal prognosis. However, further study in larger cohorts with complete clinical data is necessary to better define the true extent and clinical implications of this interaction between postpartum diagnosis and increased risk of breast cancer recurrence and death.

Another aim of our study was to identify a methodological reason for the disparate results in prior PABC studies. An important distinction of our study is the utilization of rigorously defined parity status. Prior studies have combined pregnant and early postpartum cases as PABC and utilized various referent populations. Six published studies reporting outcomes included parous women diagnosed within seven to 24 months of last childbirth in the non-PABC "control" group-four studies identify an increased risk for PABC cases $[1,15,16,18]$ and two report no increase in risk $[11,12]$. Of the four studies identifying an increased risk for PABC cases, three used a PABC cohort comprised of predominately early postpartum cases $[1,15,18]$. One study used a PABC cohort comprised of $40 \%$ early postpartum and $60 \%$ pregnant cases [16].When we applied similar definitions of PABC [pregnant and up to 1 year postpartum] and non-PABC [nulliparous and beyond 1 year postpartum] to our cohort, we also did not observed a significant increased risk associated with PABC (Table 4). We believe the inclusion
Table 5 Clinical characteristics of cases diagnosed during pregnancy $(n=24)$

\begin{tabular}{lc}
\hline & $N(\%)$ \\
\hline Histologic subtype & \\
Ductal & $22(91.7)$ \\
Lobular & $1(4.16)$ \\
Missing & $1(4.16)$ \\
Histologic grade & \\
Grade I & $0(0)$ \\
Grade II & $5(20.8)$ \\
Grade III & $17(70.8)$ \\
Missing & $2(8.33)$ \\
Lymph node involvement \\
Positive & $15(62.50)$ \\
Negative & $8(33.33)$ \\
Missing & $1(4.16)$ \\
Stage & \\
0 & $0(0)$ \\
I & $5(20.83)$ \\
II & $9(37.50)$ \\
III & $6(25.00)$ \\
IV & $4(16.67)$ \\
Biologic subtype & \\
Luminal A & $5(20.80)$ \\
Luminal B & $5(20.80)$ \\
Her2 neu positive & $5(20.80)$ \\
Triple negative & $6(25.00)$ \\
Missing & $3(12.6)$ \\
Lymphovascular invasion \\
Present & $6(25.00)$ \\
Absent & $9(37.50)$ \\
Missing & $9(37.50)$ \\
Tumor size & \\
M.1- $\leq 2.0$ cm & $9(37.50)$ \\
$>2.0-5.0$ cm & $9(37.50)$ \\
\hline & $3(12.50)$ \\
Missing & \\
\hline
\end{tabular}

of high-risk postpartum cases in the non-PABC control population blurs the true risk associated with a postpartum and nulliparous diagnosis. While our numbers of combined pregnant and $<1$ year postpartum are small, we provide evidence for the first time that the definition of the PABC and the nulliparous groups may obscure the risk of metastasis and death in postpartum breast cancer.

Currently, it is challenging to explain the conflicting data provided by these numerous studies to clarify the potential individual contributions of the pregnant and postpartum settings on breast cancer outcomes. Studies with pregnancy-specific data often include cases definitively treated during pregnancy, cases with early trimester terminations, and cases diagnosed during pregnancy, but treated postpartum $[12,18,32]$ within the pregnant cohort. 


\section{Identifying the frequency of postpartum} PABC in this Colorado cohort

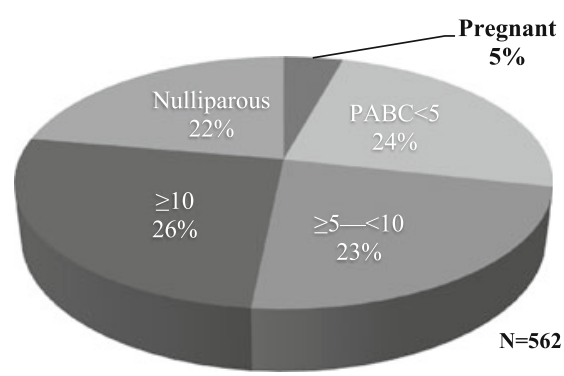

Fig. 3 Expanding the definition of $\mathrm{PABC}$ as cases diagnosed within five-years postpartum, we demonstrate $29 \%$ of cases have an increased risk for poor prognosis. Only $10 \%$ are considered PABC when defined as cases pregnant and postpartum up to one year

The impact of these clinical factors on breast cancer outcomes for women diagnosed during pregnancy is unknown. Furthermore, studies examining pregnant cases treated with definitive intent during pregnancy and concomitant maintenance of the pregnancy demonstrate equivalent outcomes to age-matched, stage-matched, non-pregnant controls [33, 34]. Our pregnancy cohort all completed delivery and received surgery and/or chemotherapy during pregnancy. We report a higher percentage of Her2 neu and triple negative cases over other subtypes which are unique to cases diagnosed during pregnancy (Table 5). A similar increase of triple negative associated with diagnosis during pregnancy was recently reported [17]. However, the size of our pregnant cohort does not permit further characterization, conclusions, or outcomes investigation at this time. Currently, others are making important efforts to build a large pregnant breast cancer registry to provide the resources necessary to help address these questions [2].

Our observation of equal presentation of biologic subtypes between nulliparous, $\mathrm{PABC}<5$, and later parous cases contrasts prior reports $[1,12,13,16,17]$ and may reflect underlying differences in sociodemographic and lifestyle factors in this dominantly Caucasian, single-state cohort. Importantly, it may also reflect a difference in outcomes depending on the definition of PABC. Previous studies that reported on combined pregnant and early postpartum cancers found an "aggressive phenotype," characterized by advanced clinical stage, larger tumor size, increased nodal involvement, and higher histologic grade $[1,11,12,16]$. Moreover, compared to non-PABC cases, various studies reported these combined pregnant and early postpartum tumors are marked by reduced estrogen and progesterone receptor positivity $[1,12,13,16]$, as well as higher frequency of Her2 neu positivity but not specifically the triple negative subtype [13, 17]. When early postpartum and pregnant cases were evaluated independently, there was contradictory data reported on the clinicopathologic features of these pregnant and postpartum subsets $[12,13$, 17-20]. Moreover, a recent PABC publication concluded triple negative tumors were more likely to be diagnosed within two-years postpartum [17]. However, in that study, when the pregnant $(n=8)$ cases were separated from the exclusively postpartum cases $(n=30)$, there was no association of triple negative tumors with a postpartum diagnosis [17]. In summary, we believe it is important to recognize that the characteristics of breast cancers diagnosed during pregnancy or postpartum may differ based on PABC definition, as well as due to potential confounders such as race or size of the cohort. The causal relationships between concurrent or prior pregnancy and tumor characteristics at diagnosis are not fully understood and beyond the scope of this report. However, as our data demonstrate, tumor characteristics alone do not account for the poorer prognosis of postpartum breast cancer and identification of the drivers for this increased risk of postpartum breast cancers are necessary to improve clinical outcomes.

To our knowledge, our study is unique in providing a contemporary comparison of outcomes between exclusively nulliparous and postpartum young women's breast cancer cohorts. The favorable outcomes of our nulliparous group, compared to prior studies with similar cohorts, may reflect a period effect as breast cancer treatment has improved significantly over time [24, 31, 32]. Our data demonstrate a striking 2.7 higher risk of death for women diagnosed within five-years postpartum compared to nulliparous, after adjusting for biologic subtype, clinical stage, and year of diagnosis. Our research program has identified postpartum breast involution as being promotional to tumor growth, invasion, and metastasis in preclinical models [30]. Similar tumor promotional attributes have been identified in human breast involution [30,35], as recently reviewed [36]. Therefore, we hypothesized that human postpartum breast cancer has poorer prognosis that is not solely explained by an enrichment of traditional poor prognostic factors that are tumor cell centric $[8,10,30,35]$. While our results do not definitively prove the role of involution as etiologic for poorer postpartum outcomes, it does support the potential that breast involution imparts a unique tumor microenvironment as a mechanism for this increased risk $[8,36]$. Further research in human cohorts on the interaction of pregnancy and subsequent breast cancer will need to include additional potential confounders, such as diversification of race, number of prior pregnancies with age at first pregnancy and intervals between, lactation data, gene mutation status, and detailed family history.

The under recognition of $\mathrm{PABC}$ and its impact on maternal outcomes is likely an unintended consequence of the variable definitions of the disease and the conflicting outcomes data. We demonstrate that limiting the definition 
of PABC as cases diagnosed during or within one year of a completed pregnancy translate into a low PABC incidence (10\%) in our Colorado cohort. Expanding the definition of PABC to within five-years postpartum increases this incidence to $29 \%$ for women diagnosed $\leq 45$ years. This higher PABC incidence is substantiated in a recently published study of young women's breast cancer where $35 \%$ of cases were diagnosed within five-years postpartum [17]. In addition, PABC incidence could be as high as $53.0 \%$ or more of young women's breast cancer if the trend in later parous groups $(\geq 5$ and $<10$ years postpartum) becomes significant in analyses of larger cohorts with longer followup (Fig. 3). If the distribution of postpartum $\mathrm{PABC}$ in our cohort is nationally representative, over a five-year period we would expect approximately 34,000 incident invasive breast cancer cases attributable to high risk postpartum PABC $(\mathrm{PABC}<5)$, and 6,900 incident diagnoses annually [37, 38].

Pregnancy ubiquitously proceeds involution and has its own significant biologic programs that influence breast cancers diagnosed both ante- and postpartum [8, 36]. Postpartum breast involution is a biologically unique event that is also emerging as a potential risk factor for premenopausal breast cancer [8, 30, 36]. Understanding the differences between breast cancers diagnosed during pregnancy and postpartum would better permit the translation of informative data from basic science and epidemiologic studies into the clinical care of young women's breast cancer. Therefore, we propose defining PABC as two distinct subsets-cases diagnosed during pregnancy and cases diagnosed postpartum - to facilitate clarity in the PABC field.

Acknowledgments We thank Betsy Risendall, PhD, Assistant Research Professor, Department of Community and Behavior Health, The Colorado School of Public Health, University of Colorado Cancer Center, University of Colorado Denver Anschutz Medical Campus, and Anna E. Baron, PhD, Professor, Department of Community and Behavior Health, Department of Biostatistics and Informatics, The Colorado School of Public Health, University of Colorado Denver Anschutz Medical Campus, for Design and conduct of the study. We also thank Amy Kendall, CTR, Lead Medical Registrar, Cancer Registry, University of Colorado Hospital, for her help in Data collection and Ann D. Thor, MD, Department Chair, Department of Pathology, University of Colorado Denver Anschutz Medical Campus, for Research Assistant support.

Funding/Support Department of Defense BC060531 to Pepper Schedin and Virginia Borges, AACR-BCRF 09-60-26 to Virginia Borges, Avon Foundation to Pepper Schedin, Men for the Cure Foundation to Virginia Borges, and ACS 116056-PF-08-257-01-CSM to Traci Lyons, Grohne Family Foundation Grant to Virginia Borges and Pepper Schedin, and Glass Family Foundation to Virginia Borges.

Conflict of interest The authors declare that they have no conflict of interest.

Ethical standards Conduct of this study at the University of Colorado Hospital and the Shaw Cancer Center was performed in compliance with the standards of the Colorado Research Ethics Board (COMIRB).

Open Access This article is distributed under the terms of the Creative Commons Attribution Noncommercial License which permits any noncommercial use, distribution, and reproduction in any medium, provided the original author(s) and the source are credited.

\section{References}

1. Rodriguez AO et al (2008) Evidence of poorer survival in pregnancy-associated breast cancer. Obstet Gynecol 112(1):71-78

2. Cardonick E et al (2010) Breast cancer during pregnancy: maternal and fetal outcomes. Cancer J 16(1):76-82

3. Pregnancy and Breast Cancer (2011); Available from: http://www. cancer.org/Cancer/BreastCancer/MoreInformation/pregnancy-andbreast-cancer. Accessed 12 May 2011

4. Janerich DT, Hoff MB (1982) Evidence for a crossover in breast cancer risk factors. Am J Epidemiol 116(5):737-742

5. Lambe $\mathrm{M}$ et al (1994) Transient increase in the risk of breast cancer after giving birth. N Engl J Med 331(1):5-9

6. Lord SJ et al (2008) Breast cancer risk and hormone receptor status in older women by parity, age of first birth, and breastfeeding: a case-control study. Cancer Epidemiol Biomarkers Prev 17(7):1723-1730

7. Liu Q et al (2002) Transient increase in breast cancer risk after giving birth: postpartum period with the highest risk (Sweden). Cancer Causes Control 13(4):299-305

8. Lyons TR, Schedin PJ, Borges VF (2009) Pregnancy and breast cancer: when they collide. J Mammary Gland Biol Neoplasia 14(2):87-98

9. Rosner B, Colditz GA (1996) Nurses' health study: log-incidence mathematical model of breast cancer incidence. J Natl Cancer Inst 88(6):359-364

10. Schedin P (2006) Pregnancy-associated breast cancer and metastasis. Nat Rev Cancer 6(4):281-291

11. Murphy CG et al (2011) Current or recent pregnancy is associated with adverse pathologic features but not impaired survival in early breast cancer. Cancer 118(13):3254-3259

12. Beadle BM et al (2009) The impact of pregnancy on breast cancer outcomes in women $<$ or $=35$ years. Cancer 115(6):1174-1184

13. Genin AS et al (2012) Pregnancy-associated breast cancers: do they differ from other breast cancers in young women? Breast 21(4):550-555

14. Petrek JA, Dukoff R, Rogatko A (1991) Prognosis of pregnancyassociated breast cancer. Cancer 67(4):869-872

15. Ali SA et al (2012) Survival outcomes in pregnancy associated breast cancer: a retrospective case control study. Breast J 18(2):139-144

16. Bonnier $P$ et al (1997) Influence of pregnancy on the outcome of breast cancer: a case-control study. Societe Francaise de Senologie et de Pathologie Mammaire Study Group. Int J Cancer 72(5):720-727

17. Pilewskie M et al (2011) Association between recency of last pregnancy and biologic subtype of breast cancer. Ann Surg Oncol 19(4):1167-1173

18. Mathelin C et al (2008) Pregnancy and post-partum breast cancer: a prospective study. Anticancer Res 28(4C):2447-2452

19. Gentilini $O$ et al (2005) Breast cancer diagnosed during pregnancy and lactation: biological features and treatment options. Eur J Surg Oncol 31(3):232-236

20. Reed W et al (2003) Pregnancy and breast cancer: a populationbased study. Virchows Arch 443(1):44-50

21. Azim HA Jr et al (2012) Prognosis of pregnancy-associated breast cancer: a meta-analysis of 30 studies. Cancer Treat Rev 38(7):834-842 
22. Dodds L et al (2008) Relationship of time since childbirth and other pregnancy factors to premenopausal breast cancer prognosis. Obstet Gynecol 111(5):1167-1173

23. Bladstrom A, Anderson H, Olsson H (2003) Worse survival in breast cancer among women with recent childbirth: results from a Swedish population-based register study. Clin Breast Cancer 4(4):280-285

24. Whiteman MK et al (2004) Reproductive history and mortality after breast cancer diagnosis. Obstet Gynecol 104(1):146-154

25. Stensheim H et al (2009) Cause-specific survival for women diagnosed with cancer during pregnancy or lactation: a registrybased cohort study. J Clin Oncol 27(1):45-51

26. Johansson AL et al (2011) Increased mortality in women with breast cancer detected during pregnancy and different periods postpartum. Cancer Epidemiol Biomarkers Prev 20(9):1865-1872

27. Pike MC et al (1983) 'Hormonal' risk factors, 'breast tissue age' and the age-incidence of breast cancer. Nature 303(5920):767-770

28. Keller KB, Lemberg L (2005) Estrogen plus progestin, benefits and risks: the "Women's Health Initiative" trials. Am J Crit Care 14(2): $157-160$

29. McDaniel SM et al (2006) Remodeling of the mammary microenvironment after lactation promotes breast tumor cell metastasis. Am J Pathol 168(2):608-620
30. Lyons TR et al (2011) Postpartum mammary gland involution drives progression of ductal carcinoma in situ through collagen and COX-2. Nat Med 17(9):1109-1115

31. Daling JR et al (2002) The relation of reproductive factors to mortality from breast cancer. Cancer Epidemiol Biomarkers Prev 11(3):235-241

32. Guinee VF et al (1994) Effect of pregnancy on prognosis for young women with breast cancer. Lancet 343(8913):1587-1589

33. Garcia-Manero M et al (2009) Pregnancy associated breast cancer. Eur J Surg Oncol 35(2):215-218

34. Keleher AJ et al (2002) Multidisciplinary management of breast cancer concurrent with pregnancy. J Am Coll Surg 194(1):54-64

35. O'Brien J et al (2010) Alternatively activated macrophages and collagen remodeling characterize the postpartum involuting mammary gland across species. Am J Pathol 176(3):1241-1255

36. Kobayashi $\mathrm{S}$ et al (2012) Reproductive history and breast cancer risk. Breast Cancer 19(4):302-308

37. US Cancer Statistics: An interactive Atlas (2009). Available from: http://apps.nccd.cdc.gov/DCPC_INCA/DCPC_INCA.aspx. Accessed 13 Feb 2013

38. Proabablity of Breast Cancer in American Women (2012). Available from: http://www.cancer.gov/cancertopics/factsheet/ detection/probability-breast-cancer. Accessed 13 Feb 2013 